\title{
Electron Transfer Dissociation of Amide Nitrogen Methylated Polypeptide Cations
}

\author{
David M. Crizer and Scott A. McLuckey \\ Department of Chemistry, Purdue University, West Lafayette, Indiana, USA
}

\begin{abstract}
Unmodified and amide nitrogen methylated peptide cations were reacted with azobenzene radical anions to study the utility of electron transfer dissociation (ETD) in analyzing $\mathrm{N}$-methylated peptides. We show that methylation of the amide nitrogen has no deleterious effects on the ETD process. As a result, location of alkylation on amide nitrogens should be straightforward. Such a modification might be expected to affect the ETD process if hydrogen bonding involving the amide hydrogen is important for the ETD mechanism. The partitioning of the ion/ion reaction products into all of the various reaction channels was determined and compared for modified and unmodified peptide cations. While subtle differences in the relative abundances of the various ETD channels were observed, there is no strong evidence that hydrogen bonding involving the amide nitrogen plays an important role in the ETD process. (J Am Soc Mass Spectrom 2009, 20, 1349-1354) (c) 2009 American Society for Mass Spectrometry
\end{abstract}

$\mathrm{T}$ andem mass spectrometry has become an essential tool in proteomics and other protein-related measurements [1]. Most applications of tandem mass spectrometry in this area involve collisional activation of polypeptide cations [2]. While this approach has proven to be effective in many scenarios, a major drawback is the cleavage of labile post-translational modifications (PTMs). Recently, electron capture and electron transfer dissociation (ECD and ETD) have proven to be useful techniques for peptide and protein structural characterization, in part because these methods often succeed in characterizing modified peptides and proteins in cases where collisional activation does not [3-8]. While ETD has been used to investigate many PTMs, little has been studied on the application of ETD to peptide and protein ions modified on the backbone.

Methylation of nitrogen can occur wherever there is a nitrogen atom, such as in lysine and arginine residues, as well as on the amide nitrogen in peptide and protein backbones. ECD [9-12] and ETD [13] have already been shown to be effective in identifying side-chain and $\mathrm{N}$-terminal methylation in histones, for example. Amide nitrogen methylation occurs in nature, such as in bacterial and fungal peptides/proteins that have possible medicinal properties [14-17], and has been used as a tool for protein binding studies [18]. The dissociation behavior of N-methylated peptide ions subjected to collisional activation has been reported, and it was noted that there was an increase in relative abundance of the products coming from cleavage of the

Address reprint requests to Dr. S. McLuckey, Department of Chemistry, Purdue University, West Lafayette, IN 47907-2084, USA. E-mail: mcluckey@ purdue.edu amide bond at the methylation site $[19,20]$. It was suggested that this preferred cleavage occurred due to the increased basicity of the amide nitrogen. In this work, we have examined how N-methylated protonated peptides behave when they are subjected to the ETD process.

In addition to its relevance to the characterization of modified proteins, this work may also have significance from a mechanistic point-of-view. While ECD and ETD have been very useful in a variety of applications, questions remain regarding various aspects of the mechanism(s) of the processes. For example, a difference in the various proposed mechanisms relates to the location of electron attachment in peptide cations. McLafferty and coworkers were the first to suggest a mechanism for this process [4]. They proposed that an electron attaches to a protonated side-chain group producing a radical species. Transfer of a hydrogen atom to the amide carbonyl can then occur to give an aminoketyl radical. This intermediate can then readily undergo dissociation at the $\mathrm{N}-\mathrm{C}_{\alpha}$ bond to give rise to $\mathrm{c}-$ and z-type ions. An alternative to this mechanism has been proposed independently by Turecek and Simons [2123]. In this picture, it is posited that electron capture occurs either directly in amide $\pi^{*}$ orbitals that are stabilized by the electrostatic field present in the multiply charged ion, or an electron can transfer to such an orbital from a low-lying Rydberg state. The population of an amide $\pi^{*}$ orbital destabilizes the $\mathrm{N}-\mathrm{C}_{\alpha}$ bond and increases dramatically the basicity of the amide group. Cleavage of the $\mathrm{N}-\mathrm{C}_{\alpha}$ bond can occur either after proton transfer to the site from elsewhere in the molecule, thereby producing the aminoketyl radical, or fragmentation of the $\mathrm{N}-\mathrm{C}_{\alpha}$ bond can occur first with either subsequent proton transfer to a fragment or 
stabilization of the fragment with the excess charge by charged groups elsewhere in the molecule. The latter scenario can account for the fact that ETD and ECD has been noted to occur in ions with fixed charges (i.e., in species for which proton transfer is unlikely).

Recently, a mechanism has been presented that places an emphasis on the importance of neutral hydrogen bonding between the amide nitrogen atom and a backbone carbonyl in the ECD/ETD process [24]. In this mechanism, it is proposed that an electron is captured by an $\mathrm{N}-\mathrm{H} \ldots \mathrm{O}=\mathrm{C}$ group forming an anionic group on the nitrogen. A proton can then be transferred to this site generating an aminoketyl radical intermediate that subsequently fragments at the $\mathrm{N}-\mathrm{C}_{\alpha}$ bond to give the observed $\mathrm{c}$ and $\mathrm{z}$ ions.

In this study, we compare the ETD behavior of two sets of model peptides, with and without methylated amide nitrogen groups. The partitioning of products among all the possible competing processes is examined closely to study the role of amide nitrogen methylation on the competition between electron-transfer and proton transfer, the tendency for electron-transfer to result in spontaneous fragmentation (i.e., ETD) versus electrontransfer without dissociation, and possible differences in partitioning between the various available fragmentation channels.

\section{Experimental}

Nanospray emitters were pulled from borosilicate capillaries (1.55 mm o.d., $0.86 \mathrm{~mm}$ i.d.) using a P-87 Flaming/Brown micropipet puller (Sutter Instruments, Novato, CA). These nanospray tips were loaded with the electrospray solutions, and a metal wire was inserted into the tip to make electrical contact with the solution [25].

Ionization was performed using a home-built pulsed dual ionization (nano-ESI and APCI) source that has been previously described [26]. This pulsed dual source consists of one nano-ESI emitter for the generation of highly charged peptide cations and an atmospheric pressure chemical ionization (APCI) needle for the formation of azobenzene radical anions, which were pulsed on and off in each scan by the software. High voltages applied to the emitters were optimized separately and were independently generated by two highvoltage power supplies, one from the instrument and one from an external supply gated by a fast high voltage pulser (GRX-1.5K-E; Directed Energy Inc., Fort Collins, $\mathrm{CO})$. Using this method, ions of either polarity could be independently optimized and directed into the mass spectrometer.

A quadrupole/time-of-flight tandem mass spectrometer (QSTAR XL; Applied Biosystems/MDS SCIEX, Concord, ON, Canada) that has been modified for ion/ion reactions was used for all experiments [27]. Nitrogen was used as the collision gas. The instrument was controlled by a research version of Daetalyst (MDS SCIEX), which provided full control for initiating each ion source and for applying the appropriate potentials and their timing to the ion path.

A typical experiment consisted of producing peptide cations via the positive nano-ESI source and isolating the ions of interest as they passed through Q1. The isolated cations were then directed into the Q2 linear ion trap (LIT) (200 ms), where they were trapped. The azobenzene reagent ions were produced via atmospheric pressure chemical ionization (APCI) and were then isolated in Q1, and introduced into the Q2 LIT (100 $\mathrm{ms})$, where ions of both polarities were mutually stored and allowed to react $(50 \mathrm{~ms})$. The ion/ion reaction products were then mass analyzed by a reflectron time-of-flight (TOF) analyzer (150 ms). The spectra shown here were typically averages of 100-200 scans. Data shown were normalized to the most abundant product ion.

The peptides KAAAKAAAK, KGGGKGGGK, and their N-methylated analogs were synthesized by CPC Scientific (San Jose, CA) and used without further purification. The peptides were diluted to a concentration of 0.1 $\mathrm{mg} / \mathrm{mL}$ in 49/49/2 (vol/vol/vol) methanol/water/acetic acid solution.

\section{Results and Discussion}

A useful approach to identifying the effects of variables in the ETD of polypeptides is to compare the partitioning of ion/ion reaction products among all of the relevant reaction channels [28]. Specifically, this entails measurement of the relative contributions of proton transfer versus electron-transfer (PT versus ET), the partitioning between electron-transfer without dissociation (ET,noD) and with dissociation (ETD) within the overall ET channel, the partitioning between the products of backbone cleavages (e.g., the c- and z-type ions) and products from side-chain cleavages within the ETD channel, and the partitioning between the various backbone cleavages channels (i.e., specific c- and z-type ions). It is desirable to compare product partitioning at these various levels because the major effect of a particular variable in an ETD experiment, such as the nature of the reagent anion [29] or the nature of the charge bearing sites on the peptide [22], may be more manifest in one of the levels of partitioning than another. The partitioning of products is related on a percentage basis with the following relationships:

$$
\begin{aligned}
& 100 \%=\% \mathrm{PT}+\% \mathrm{ET} \\
& \% \mathrm{ET}=\% \mathrm{ETD}+\% \mathrm{ET}, \mathrm{noD} \\
& 100 \%=\% \mathrm{c}, \mathrm{z} / \mathrm{ETD}+\% \text { side-chain } / \mathrm{ETD}
\end{aligned}
$$

Table 1 summarizes the product partitioning noted for the $[\mathrm{M}+3 \mathrm{H}]^{3+}$ and $[\mathrm{M}+2 \mathrm{H}]^{2+}$ ions derived from the modified and unmodified versions of the model peptides KGGGKGGGK and KAAAKAAAK. Perhaps the most remarkable observation is that there is no clear 
Table 1. Ion/ion reaction partitioning for the reactions between peptide anions and azobenzene anions

\begin{tabular}{|c|c|c|c|c|c|c|c|c|}
\hline & $\begin{array}{c}3+ \\
\text { Unmodified } \\
\mathrm{KG}_{3} \mathrm{KG}_{3} \mathrm{~K}\end{array}$ & $\begin{array}{c}3+ \\
\text { Modified } \\
\mathrm{KG}_{3} \mathrm{KG}_{3} \mathrm{~K} \\
\end{array}$ & $\begin{array}{c}2+ \\
\text { Unmodified } \\
\mathrm{KG}_{3} \mathrm{KG}_{3} \mathrm{~K}\end{array}$ & $\begin{array}{c}2+ \\
\text { Modified } \\
\mathrm{KG}_{3} \mathrm{KG}_{3} \mathrm{~K} \\
\end{array}$ & $\begin{array}{c}3+ \\
\text { Unmodified } \\
\mathrm{KA}_{3} \mathrm{KA}_{3} \mathrm{~K}\end{array}$ & $\begin{array}{c}3+ \\
\text { Modified } \\
\mathrm{KA}_{3} \mathrm{KA}_{3} \mathrm{~K} \\
\end{array}$ & $\begin{array}{c}2+ \\
\text { Unmodified } \\
\mathrm{KA}_{3} \mathrm{KA}_{3} \mathrm{~K}\end{array}$ & $\begin{array}{c}2+ \\
\text { Modified } \\
\mathrm{KA}_{3} \mathrm{KA}_{3} \mathrm{~K} \\
\end{array}$ \\
\hline \%PT & $36 \pm 1$ & $26 \pm 1$ & $48 \pm 1$ & $52 \pm 1$ & $30 \pm 1$ & $44 \pm 1$ & $59 \pm 1$ & $53 \pm 1$ \\
\hline$\%$ ET & $64 \pm 1$ & $74 \pm 1$ & $52 \pm 1$ & $48 \pm 1$ & $70 \pm 2$ & $56 \pm 2$ & $41 \pm 1$ & $47 \pm 1$ \\
\hline$\%$ ETD & $64 \pm 1$ & $74 \pm 1$ & $52 \pm 1$ & $48 \pm 1$ & $70 \pm 2$ & $56 \pm 2$ & $41 \pm 1$ & $47 \pm 1$ \\
\hline$\%$ ET,noD & 0 & 0 & 0 & 0 & 0 & 0 & 0 & 0 \\
\hline$\% c, z / E T D$ & $81 \pm 2$ & $87 \pm 1$ & $94 \pm 1$ & $84 \pm 1$ & $86 \pm 1$ & $83 \pm 1$ & $89 \pm 1$ & $78 \pm 1$ \\
\hline$\%$ side-chain/ETD & $19 \pm 2$ & $13 \pm 1$ & $6 \pm 1$ & $16 \pm 1$ & $14 \pm 1$ & $17 \pm 1$ & $11 \pm 1$ & $22 \pm 1$ \\
\hline
\end{tabular}

and consistent difference between the product partitioning for the modified and unmodified species. For example, the modified version of triply protonated KGGGKGGGK shows a slightly greater tendency for electron-transfer than the unmodified version, whereas the opposite situation is observed for the modified and unmodified versions of KAAAKAAAK. In all cases, essentially all peptide ions that underwent electrontransfer, regardless of charge or modification state, went on to fragment. This is consistent with studies of other model peptides in which the likely sites of protonation are primary amines (i.e., lysine side chains or N-termini). Similar partitioning between dissociation channels that lead to c- and/or z-type ions and sidechain losses were also noted for the modified and unmodified versions. This set of observations suggests that the amide nitrogen methylation plays little direct role in the ETD process. The competition between PT and ET is not expected to be particularly sensitive to $\mathrm{N}$-methylation because the main cation characteristic

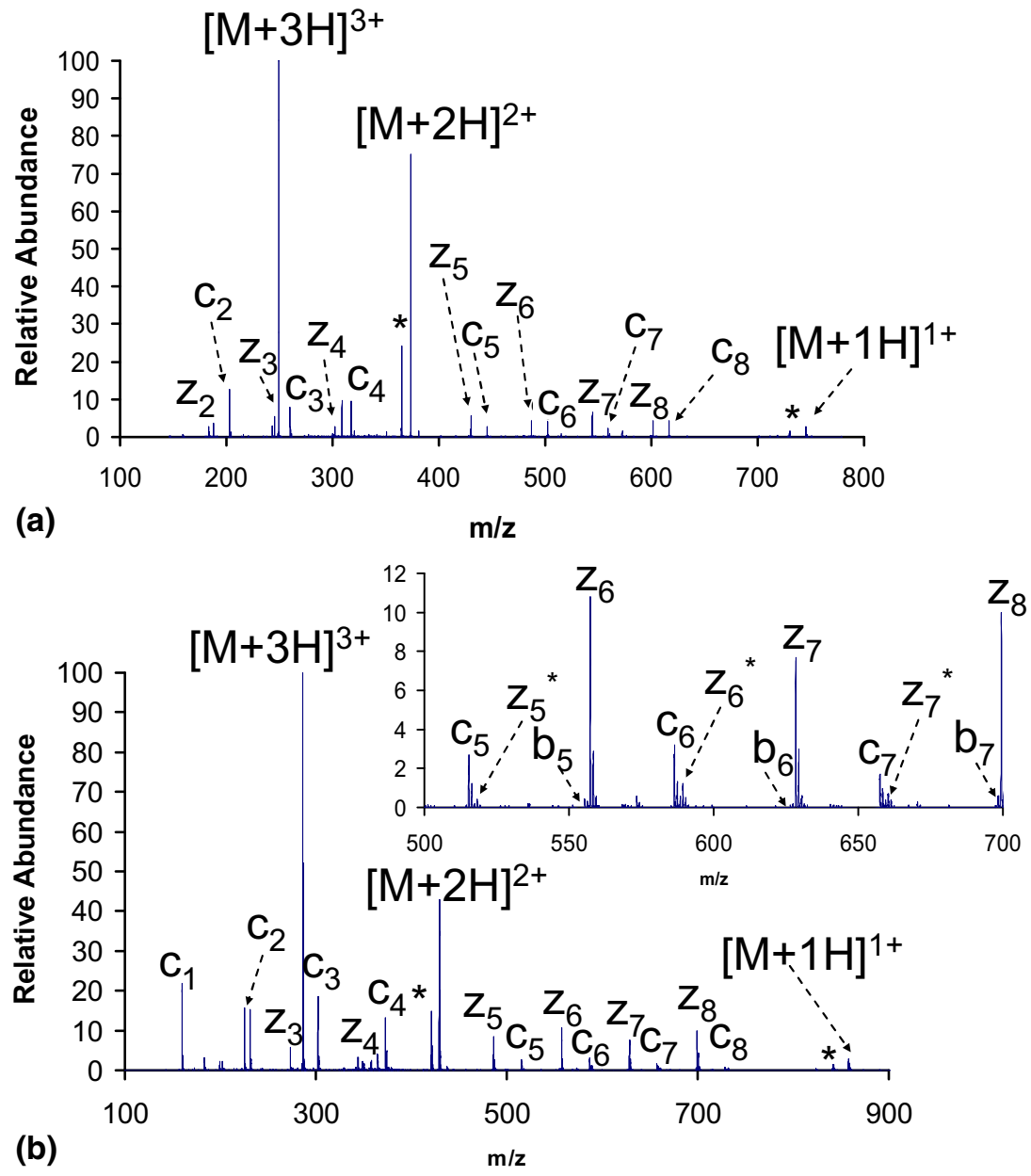

Figure 1. Spectra from the reaction of azobenzene radical anions with triply charged (a) KGGGKGGGK and (b) N-methylated KGGGKGGGK, where the asterisk stands for $\mathrm{NH}_{3}$ loss. The inset is an expanded view of the region from $\mathrm{m} / \mathrm{z} 500$ to 700, where $\mathrm{z}^{*}$ represents $\mathrm{O}_{2}$ adducts. 
that affects this competition is the recombination energy of the cation [23], which is not expected to change significantly upon $\mathrm{N}$-methylation of the model peptides. However, if the $\mathrm{N}-\mathrm{H} \ldots \mathrm{O}=\mathrm{C}$ interaction is important for generating $\mathrm{c}$ - and z-ions, the elimination of this interaction via N-methylation might be expected to affect the competition between ETD and ET,noD and almost certainly the competition between side-chain cleavage and $\mathrm{c} / \mathrm{z}$-ion formation.

It is also of interest to examine the role of $\mathrm{N}$ methylation on the partitioning among the various backbone dissociation channels. The ETD spectra for reactions between the azobenzene molecular anion and the triply charged unmodified and modified versions of KGGGKGGGK are shown in Figure 1. Both the modified and unmodified species show complete sequence coverage. Similarly, when doubly charged peptide ions were reacted with azobenzene anions, complete sequence coverage also resulted (see Figure 2). The spectra of the doubly charged precursor ions do not show complementary c- and z-type ions, as do the spectra of the triply charged precursor ions, because only one of the complementary fragments from the doubly protonated precursor can carry a charge. In all cases, the higher mass fragment was favored to carry the excess charge upon ETD of the doubly protonated precursor ion.

The plots of Figure 3 summarize differences in the partitioning among the various ETD channels for the unmodified and modified versions of the two charge states of each of the two model systems examined. The plots were generated by normalizing the total ETD product signal in each spectrum to 100 and subtracting the normalized product abundances for each channel derived from the modified ion of a given charge state from the unmodified version. Bars present above the zero line indicate that a particular pathway contributes more on a relative basis to the ETD spectra of the unmodified peptides, while bars below the zero line indicate that the pathway is relatively more favorable in the ETD spectra of the modified peptides. The data show that there are measurable differences in the partitioning among the various backbone dissociation channels for the modified and unmodified peptides. However, the
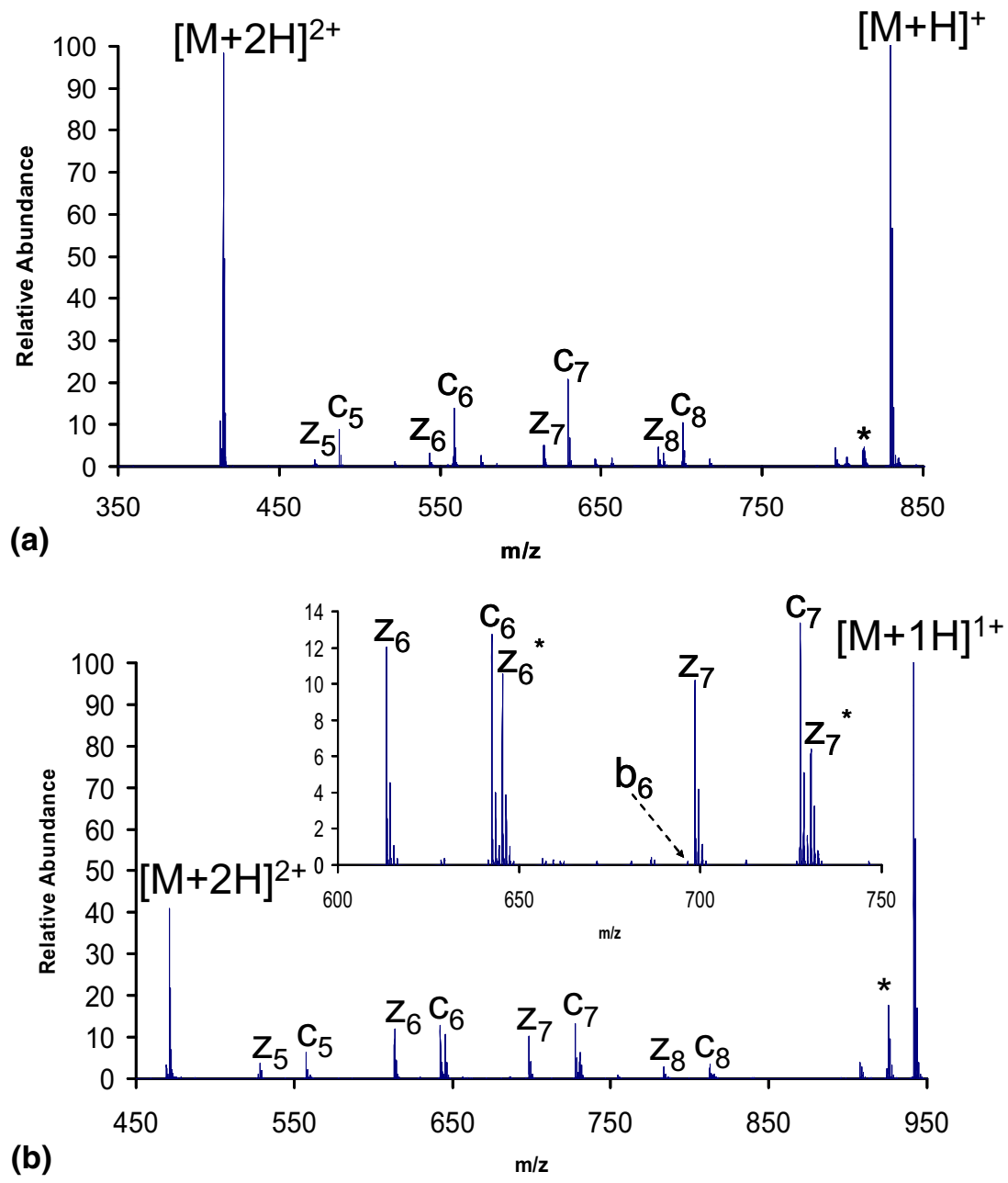

Figure 2. Spectra from the reaction of azobenzene radical anions with doubly charged (a) KAAAKAAAK and (b) N-methylated KAAAKAAAK, where the asterisk stands for $\mathrm{NH}_{3}$ loss. The inset shows an expanded view from $\mathrm{m} / \mathrm{z} 600$ to 750 , where $\mathrm{z}^{*}$ stands for $\mathrm{O}_{2}$ adducts. 

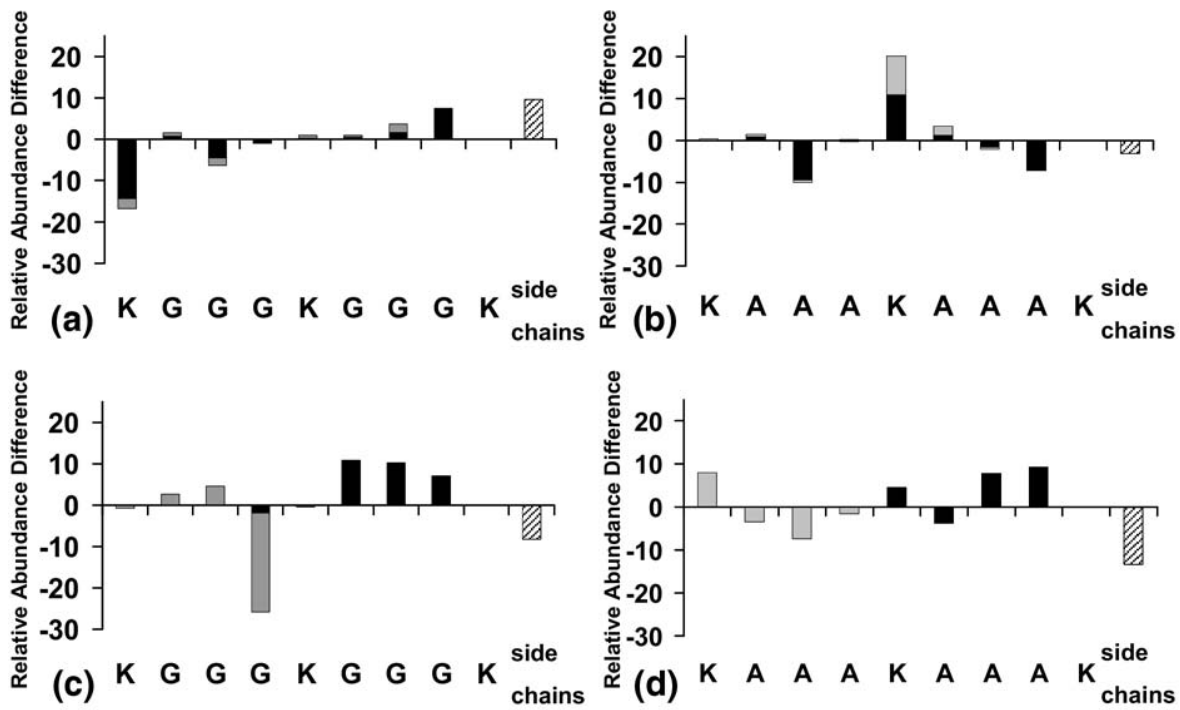

Figure 3. Difference plots comparing differences between ETD spectra of modified peptides subtracted from unmodified peptides (a) triply charged KGGGKGGGK, (b) triply charged KAAAKAAAK, (c) doubly charged KGGGKGGGK, and (d) doubly charged KAAAKAAAK; where black, grey, and striped bars represent c- ions, z- ions, and side-chain losses, respectively.

differences are not dramatic, and there appear to be no clear tendency for the four cases examined (e.g., no individual dissociation channel is favored for either the modified or unmodified form for all of the cases). The differences that are observed may very well arise from conformational effects associated with backbone methylation, although no definitive conclusions can be drawn.

While the overall ETD behaviors of the modified and unmodified peptide cations were similar, a few additional products at low abundance were observed in the data for the modified species that were either absent or far less abundant in the data for the unmodified ions. For example, many of the z-ions showed a small peak 2 Da lower in mass. These ions match the predicted masses of $b$-ions. The presence of $b$ ions in ETD/ECD spectra has been previously discussed [30, 31]. However, in this case, a more likely origin of the b-ions is from collisional activation of the precursor ion upon injection into the collision cell. The CID study of methylated peptides showed favored cleavage at the modification site, which indicates that $\mathrm{N}$-methylation reduces the stability of the amide bond to cleavage. We found via CID experiments (data not shown) that $b$ ions are the first products noted near threshold. Another set of products was consistently observed $3 \mathrm{Da}$ higher in mass than the c-ions. These ions are consistent in mass with oxygen adducts to z-ions, an ion/molecule reaction that has recently been reported [32]. Interestingly, much lower relative abundances of $z+32$ ions were observed in the data for the unmodified peptide ions, which implies that the radical z-type ions generated from the N-methylated species, which may be comprised of a mixture of structures, are more reactive with oxygen that the population of z-ions generated from the unmethylated peptide.

\section{Conclusions}

Methylation of the amide nitrogen in a peptide has little influence in the ETD of its gas-phase multiply protonated ions. The presence of a methylation site can therefore be expected to be reflected in the masses of the cand z-ions that contain the methylation site, as is the case with side-chain post-translational modifications. While the methyl modification is expected to be strongly bound, its presence on the amide nitrogen might have been expected to affect the overall ETD process, at least based on one proposed mechanism for ECD/ETD [18]. $\mathrm{N}$-methylation affects modestly the relative abundances of the sequence informative fragment ions but this observation may very well result from conformational differences between modified and unmodified versions of the peptide cations. There is no strong evidence that hydrogen bonding associated with the amide hydrogen in unmodified peptides plays a significant role in the ETD process. N-methylated peptide ions undergo CID more readily than their unmethylated counterparts and the z-type radical product ions formed via ETD appear to be more reactive to oxygen adduction than the corresponding z-ions from unmodified versions of the peptide.

\section{Acknowledgments}

The authors acknowledge support for this work by the Office of Basic Energy Sciences, Division of Chemical Sciences under Award no. DE-FG02-00ER15105. 


\section{References}

1. Han, X.; Aslanian, A.; Yates, J. R. III. Mass Spectrometry for Proteomics. Curr. Opin. Chem. Biol. 2008, 12, 1-8.

2. Wells, J. M.; McLuckey, S. A. Collision-Induced Dissociation (CID) of Peptides and Proteins. Methods Enzymol. 2005, 402, 148-185.

3. Zubarev, R. A.; Kelleher, N. L.; McLafferty, F. W. Electron Capture Dissociation of Multiply Charged Protein Cations. A Nonergodic Process. J. Am. Chem. Soc. 1998, 120, 3265-3266.

4. Zubarev, R. A.; Kruger, N. A.; Fridricksson, E. K.; Lewis, M. A.; Horn, D. M.; Carpenter, B. K.; McLafferty, F. W. Electron Capture Dissociation of Gaseous Multiply-Charged Proteins is Favored at Disulfide Bonds and Other Sites of High Hydrogen Atom Affinity. J. Am. Chem. Soc. 1999, 121, 2857-2862.

5. Zubarev, R. A.; Horn, D. M.; Fridricksson, E. K.; Kelleher, N. L.; Kruger, N. A.; Lewis, M. A.; Carpenter, B. K.; McLafferty, F. W. Electron Capture Dissociation for Structural Characterization of Multiply Charged Protein Cations. Anal. Chem. 2000, 72, 563-573.

6. Zubarev, R. A. Reactions of Polypeptide Ions with Electrons in the Gas Phase. Mass Spectrom. Rev. 2003, 22, 57-77.

7. Syka, J. E. P.; Coon, J. J.; Schroeder, M. J.; Shabanowitz, J.; Hunt, D. F. Peptide and Protein Sequence Analysis by Electron Transfer Dissociation Mass Spectrometry. Proc. Natl. Acad. Sci. U.S.A. 2004, 101, $9528-$ 9533.

8. Pitteri, S. J.; Chrisman, P.A.; Hogan, J. M.; McLuckey, S. A. Electron Transfer Ion/Ion Reactions in a Three-Dimensional Quadrupole Ion Trap: Reactions of Doubly and Triply Protonated Peptides with $\mathrm{SO}_{2}$. Anal. Chem. 2005, 77, 1831-1839.

9. Cooper, H. J.; Hudgins, R. R.; Marshall, A. G. Electron Capture Dissociation Fourier Transform Ion Cyclotron Resonance Mass Spectrometry of Cyclodepsipeptides, Branched Peptides, and e-Peptides. Int. J. Mass Spectrom. 2004, 234, 23-35.

10. Zhang, L.; Freitas, M. A. Comparison of Peptide Mass Mapping and Electron Capture Dissociation as Assays for Histone Post-Translational Modifications. Int. J. Mass Spectrom. 2004, 234, 213-225.

11. Medzihradszky, K. F.; Zhang, X.; Chalkley, R. J.; Guan, S.; McFarland, M. A.; Chalmers, M. J.; Marshall, A. G.; Diaz, R. L.; Allis, C. D.; Burlingame, A. L. Characterization of Tetrahymena Histone H2B Variants and Post-Translational Populations by Electron Capture Dissociation (ECD) Fourier Transform Ion Cyclotron Mass Spectrometry. Mol. Cell. Proteom. 2004, 3, 872-886.

12. Su, X.; Ren, C.; Freitas, M. A. Mass Spectrometry-Based Strategies for Characterization of Histones and Their Post-Translational Modifications. Exp. Rev. Proteom. 2007, 4, 211-225.

13. Taverna, S. D.; Ueberheide, B. M.; Liu, Y.; Tackett, A. J.; Diaz, R. L. Shabanowitz, J.; Chait, B. T.; Hunt, D.F.; Allis, C. D. Long-Distance Combinatorial Linkage Between Methylation and Acetylation on Histone H3 N Termini. Proc. Natl. Acad. Sci. U.S.A. 2007, 104, 2086-2091.

14. Braun, W.; Kallen, J.; Mikol, V.; Walkinshaw, M. D.; Wuthrich, K. 3-Dimensional Structure and Actions of Immunosuppressants and Their Immunophilins. FASEB J. 1995, 9, 63-72.

15. Seebach, D.; Studer, A.; Pfammatter, E.; Widmer, H. Synthesis of Tripeptides, Pentapeptides, and Heptapeptides Containing an (R)-2Alkyl-2-Amino-3-(Methylamino)-Propionic Acid Residue in the Central Position. Helv. Chim. Acta 1994, 77, 2035-2050.
16. Fusetani, N.; Matsunaga, S. Bioactive Sponge Peptides. Chem. Rev. 1993, 93, 1793-1806.

17. Muller, G.; Gurrath, M.; Kurz, M.; Kessler, H. $\beta$-Vi Turns in Peptides and Proteins - a Model Peptide Mimicry. Prot. Struct. Funct. Genet. 1993, 15, 235-251.

18. Barbier, J.-R.; Gardella, T. J.; Dean, T.; MacLean, S.; Potetinova, Z.; Whitfield, J. F.; Willick, G. E. Backbone-Methylated Analogues of the Principle Receptor Binding Region of Human Parathyroid Hormone. J. Biol. Chem. 2005, 280, 23771-23777.

19. Nair, H.; Somogyi, A.; Wysocki, V. H. Effect of Alkyl Substitution at the Amide Nitrogen on Amide Bond Cleavage: Electrospray Ionization Surface-Induced Dissociation Fragmentation of Substance P and Two Alkylated Analogs. J. Mass Spectrom. 1996, 31, 1141-1148.

20. Vaisar, T.; Urban, J. Gas-Phase Fragmentation of Protonated Mono-Nmethylated Peptides. Analogy with Solution-Phase Acid-catalyzed Hydrolysis. J. Mass. Spectrom. 1998, 33, 505-524.

21. Syrstad, E. A.; Turecek, F. Toward a General Mechanism of Electron Capture Sissociation. J. Am. Soc. Mass Spectrom. 2005, 16, 208-224.

22. Anusiewicz, W.; Berdys-Kochanska, J.; Simons, J. Electron Attachment Step in Electron Capture Dissociation (ECD) and Electron Transfer Dissociation (ETD). J. Phys. Chem. A 2005, 109, 5801-5813.

23. Sobczyk, M.; Anusiewicz, W.; Berdys-Kochanska, J.; Sawicka, A.; Skurski, P.; Simons, J. Coulomb-Assisted Dissociative Electron Attachment: Application to a Model Peptide. J. Phys. Chem. A 2005, 109, 250-25.

24. Patriksson, A.; Adams, C.; Kjeldsen, F.; Raber, J.; van der Spoel, D. Zubarev, R. A. Prediction of $\mathrm{N}-\mathrm{C}_{\alpha}$ Bond Cleavage Frequencies in Electron Capture Dissociation of Trp-Cage Dications by Force-Field Molecular Dynamics Simulations. Int. J. Mass Spectrom. 2006, 248, $124-135$.

25. Van Berkel, G. J.; Asano, K. G.; Schnier, P. D. Electrochemical Processes in a Wire-in-a-Capillary Bulk-Loaded, Nano-Electrospray Emitter. J. Am. Soc. Mass Spectrom. 2001, 12, 853-862.

26. Liang, X.; Xia, Y.; McLuckey, S. A. Alternately Pulsed Nanoelectrospray Ionization/Atmospheric Pressure Chemical Ionization for Ion/Ion Reactions in an Electrodynamic Ion Trap. Anal. Chem. 2006, 78, 3208-3212.

27. Xia, Y.; Chrisman, P. A.; Erickson, D. E.; Liu, J.; Liang, X.; Londry, F. A.; Yang, M. J.; McLuckey, S. A. Implementation of Ion/Ion Reactions in a Quadrupole/Time-of-Flight Tandem Mass Spectrometer. Anal. Chem. 2006, 78, 4146-4154.

28. Xia, Y.; Gunawardena, H. P.; Erickson, D. E.; McLuckey, S. A. Effects of Cation Charge-Site Identity and Position on Electron-Transfer Dissociation of Polypeptide Cations. I. Am. Chem. Soc. 2007, 129, 12232-12243.

29. Gunawardena, H. P.; He, M.; Chrisman, P.A.; Pitteri, S. J.; Hogan, J. M.; Hodges, B. D. M.; McLuckey, S. A. Electron Transfer Versus Proton Transfer in Gas-Phase Ion/Ion Reactions of Polyprotonated Peptides. J. Am. Chem. Soc. 2005, 127, 12627-12639.

30. Cooper, H. J. Investigation of the Presence of b Ions in Electron Capture Dissociation Mass Spectra. J. Am. Soc. Mass Spectrom. 2005, 16, 1932 1940.

31. Haselmann, K. F.; Schmidt, M. Do b-Ions Occur from Vibrational Excitation upon H-Desorption in Electron Capture Dissociation? Rapid Commun. Mass Spectrom. 2007, 21, 1003-1008.

32. Xia, Y.; Chrisman, P. A.; Pitteri, S. J.; Erickson, D. E.; McLuckey, S. A Ion/Molecule Reactions of Cation Radicals Formed from Protonated Polypeptides via Gas-Phase Ion/Ion Electron Transfer. J. Am. Chem. Soc. 2006, 128, 11792-11798. 